\title{
Acupuncture And Chinese Herb Medicine Yangjing Zhongyu Decoction For The Treatment of Endometriosis-Associated Infertility: A Study Protocol For A Multi-Center, Controlled and Randomized Clinical Trial
}

Chuting Wu ( $\nabla$ wuchutingkk@163.com )

Shanghai University of Traditional Chinese Medicine https://orcid.org/0000-0001-5528-1450

Xiaoyun Zhang shang hai zhong yi yao da xue fu shu long hua yi yuan: Long Hua Hospital

Chunyan Zhang

Shanghai University of Traditional Chinese Medicine Yueyang Hospital of Integrated Traditional Chinese Medicine and Western Medicine https://orcid.org/0000-0001-5016-2444

\section{Research Article}

Keywords: endometriosis, infertility, Traditional Chinese Medicine, Chinese herbal medicine, acupuncture, Yangjing Zhongyu Decoction

Posted Date: May 24th, 2021

DOI: https://doi.org/10.21203/rs.3.rs-402051/v1

License: (c) (1) This work is licensed under a Creative Commons Attribution 4.0 International License. Read Full License 


\title{
Acupuncture and Chinese herb medicine Yangjing Zhongyu \\ Decoction for the treatment of endometriosis-associated \\ infertility: a study protocol for a multi-center, controlled and randomized clinical trial
}

\author{
Chuting $\mathrm{Wu}^{1,2}$, Xiaoyun Zhang ${ }^{3}$, Chunyan Zhang ${ }^{1}$ \\ 1.Yueyang Hospital of Integrated Traditional Chinese and Western Medicine, \\ Shanghai University of Traditional Chinese Medicine, Shanghai, China \\ 2.Shanghai University of Traditional Chinese Medicine, Shanghai, China \\ 3.Longhua Hospital Shanghai University of Traditional Chinese Medicine, Shanghai, \\ China \\ Correspondence to Dr. Chunyan Zhang; zh_ch_yan@163.com
}

\begin{abstract}
Introduction: Endometriosis is an inflammatory estrogen-dependent gynecological disease, which is one of the common reasons for infertility. The current treatments of endometriosis-associated infertility often involve laparoscopic surgery, medical therapy and in vitro fertilization (IVF) therapy, which are expensive, and the therapeutic effects are far from the expectation. Acupuncture and Yangjing Zhongyu Decoction (YZD) which have the advantages of effective and inexpensive, have been used clinically for the infertile female in China for many years. However, a comprehensive evaluation of the current clinical evidence of their efficacy is lacking. Our study intends to evaluate the efficacy of acupuncture and YZD on endometriosis-associated infertility (EAI).
\end{abstract}

Methods/design: This study is a multi-center, controlled and randomized clinical trial. A total of 224 eligible patients with endometriosis-associated infertility will be randomly assigned into two groups, in a 1:1 ratio as the treatment group or the control group. All participants will receive pregnancy guidance. The participants assigned to the treatment group will be treated with acupuncture and YZD while the control group will receive the GnRH-a therapy. The trial will include three menstrual cycles of treatment and twelve menstrual cycles of follow-up. The primary outcome is pregnancy rate that will be verified by human chorionic gonadotropin (HCG) tests 
and secondary outcomes include the result of ultrasound, cancer antigen 125 (CA125), anti-Müllerian hormone (AMH), sex hormones, alanine aminotransferase (ALT) and the Endometriosis Health Profile-5 (EHP-5). Outcome will be collected at baseline, the end of treatments and follow-up visits at 3,6 and 12 menstrual cycles. All the data including the major adverse events will be recorded in electronic case report forms and analysed by SPSS V.25.0.

Discussion: This study protocol will help to evaluate whether acupuncture and Chinese herb medicine Yangjing Zhongyu Decoction are effective in increasing pregnancy rate of the infertile female with endometriosis.

Keywords: endometriosis, infertility, Traditional Chinese Medicine, Chinese herbal medicine, acupuncture, Yangjing Zhongyu Decoction

Trial registration: ChiCTR2100042830; Registered on 29 January 2021.

\section{Background}

As an inflammatory estrogen-dependent disease, endometriosis is characterized by the presence of endometrial glands and stroma at extrauterine sites, ${ }^{1}$ with the symptoms including severe dysmenorrhoea, abdominopelvic pain, heavy menstrual bleeding, infertility, dyspareunia, postcoital bleeding, and/or previous diagnosis of ovarian cyst and pelvic inflammatory disease. ${ }^{2}$ The prevalence of endometriosis in subfertile women is almost $50 \%$, and the incidence of infertility ranges from $30 \%$ to $50 \% .^{3,4}$ The concept of endometriosis-associated infertility (EAI) was first proposed by Buyalos et al. in 2000. ${ }^{5}$

The pathological result of laparoscopy is the gold standard for the definitive diagnosis of endometriosis. ${ }^{6}$ Meanwhile, laparoscopy is also widely used to treat endometriosis. A meta-analysis demonstrates that laparoscopic surgery can significantly improve both pregnancy rates and live birth rates in women with endometriosis, especially the women whose infertilities are primarily caused by minimal and mild endometriosis. ${ }^{7}$ Several treatments are applied to increase the pregnancy rate after the surgery, including expectant management, medical therapy, and IVF. Researchers have compared expectant management with medical therapy. Medical therapy has better efficacy in preventing relapse. ${ }^{8}$ Medications are hormonal medications, including combined oral contraceptives, progestins, danazol, and gonadotropin-releasing hormone agonists or antagonists (GnRH analogs, GnRH-a). ${ }^{9}$ 
Medical therapies are used to treat symptoms of endometriosis and eradicate residual endometriotic implants in patients with extensive disease, for whom the resection of all implants is impossible or inadvisable. Moreover, such therapies are essential in moderate and severe endometriosis, and associated infertility. Thereinto GnRH-a therapy after surgery should be considered the 'first-line' treatment. ${ }^{10-12}$ Researchers find that IVF can help women who fail to get pregnant. ${ }^{13}$ However, time consumption, high expense, side effect, and low rate of success are inevitable disadvantages. So far, no good treatments are available for EAI.

The complementary and alternative medicines, including traditional Chinese herbs, acupuncture, and moxibustion, are widely used in China. Acupuncture is an integral part of traditional Chinese medicine (TCM), which dates back about 3,000 years. Its use has also gained increasing popularity in the Western world. In the 2002 National Institutes of Health interview-survey conducted in the United States, $4.1 \%$ of the respondents have reported lifetime use, and 1.1\% (representing 2.13 million Americans) have reported recent use of acupuncture. ${ }^{14}$ Research in the United Kingdom has investigated acupuncture practitioners' experience and perceptions of supporting patients with fertility issues. Questionnaires were sent to 2,580 practitioners. Of 861 responses, $15 \%$ of practitioners supporting fertility issues said this constituted a large proportion of their case load. Practitioners' perceived benefits include stress reduction, relaxation, regulation of menstrual cycle, and emotional support. ${ }^{15}$ The use of acupuncture as a monotherapy has been proved to improve the pregnancy rate of infertile women. A meta-analysis of anovulatory infertility shows that the use of acupuncture as monotherapy significantly improves the pregnancy rate among the participants compared with the use of clomiphene citrate alone. ${ }^{16}$

Chinese herb medicine (CHM) is also widely used in endometriosis and infertility. In endometriosis, the CHM after laparoscopic surgery can increase the pregnancy rates from $40 \%$ to about $60 \% .{ }^{17,18} \mathrm{We}$ find that acupuncture and CHM are proved to be more efficient than medical therapy and CHM alone in polycystic ovarian syndrome-associated and immune infertility. ${ }^{19.20}$

Yangjing Zhongyu Decoction (YZD), which was developed by Shan Fu, a famous ancient gynecologist in China, consists of Radix rehmanniae preparata (Shudihaung), Angelica sinensis (Danggui), Radix paeoniaealba (Baishao), and Cornus officinalis (Shanzhuyu). From the theory of TCM, this prescription can 
nourish the liver and kidney, tonify the essence, and supply the blood, which is widely used in infertility.

Animal experiments have shown that YZD can adjust sex hormone levels, such as increasing the levels of estradiol testosterone and 17-hydroxyprogesterone, enhance the expressions of the follicle-stimulating hormone receptor (FSHR), insulin-like growth factor 1 (IGF-1), and steroidogenic acute regulatory protein (StAR) at the mRNA level, and promote the ovarian function and follicular developmen. ${ }^{21}$ YZD can also increase the ovulation rate, fertilization rate, cleavage rate, and blastomere normal rate, indicating that the maturation of egg cytoplasm and nucleus is promoted to enhance the quality of egg cells. ${ }^{22}$ Clinical trials have shown that YZD can not only react on the ovary but also endometrium by increasing the endometrial thickness and changing the uterine artery pulsatility index (PI) and resistance index (RI). Generally speaking, the YZD may increase the pregnancy rate in multiple ways. $^{23-25}$

Although acupuncture and YZD for improving pregnancy rates have their clinical values, the supporting evidence of EAI is limited. Therefore, we aim to investigate the clinical effectiveness of acupuncture and YZD in infertile women with endometriosis and provide solid evidence to guide clinical practice.

\section{Methods/Design}

\section{Study design}

This is a multi-center, controlled, and randomized clinical trial (RCT). A target of 224 participants will be recruited from Yueyang Hospital of Integrated Traditional Chinese and Western Medicine, Hospital for Maternity and Child Care of Changning District, and Obstetrics \& Gynecology Hospital of Fudan University. Fig. 1 illustrates the flow chart. This study complies with the Declaration of Helsinki and follows Good Clinical Practice guidelines. We will rigorously follow the latest Consolidated Standards of Reporting Trials (CONSORT 2017) for CHM recommendations, ${ }^{26}$ Revised Standards for Reporting Interventions in Clinical Trials of Acupuncture (STRICTA), ${ }^{27}$ and SPIRIT 2013 Statement: defining standard protocol items for clinical trial. ${ }^{28}$ The trial protocol is approved by the Ethics Committee of Yueyang Hospital of Integrated Traditional Chinese and Western Medicine (approval No. 2020-025). This trial is registered at the Chinese Clinical Trial Registry (ChiCTR2100042830). Any changes that need to be made in the trial protocol will be 
communicated with all investigators, the ethics committees, and the trial registry. Written informed consent is required from each participant.

\section{Inclusion criteria}

Participants who meet all the following requirements will be eligible for enrollment:

1. Women aged between 21 and 45 years with fertility intention;

2. Patients who meet the diagnostic criteria for both endometriosis and infertility; a. endometriosis: histopathological results: endometrial glands and stroma found in the lesion, accompanied by inflammation and fibrosis. ${ }^{29}$

b. infertility: women who fail to achieve a clinical pregnancy after 12 months or more of regular unprotected sexual intercourse; this includes secondary infertility which is a failure to conceive after a previous pregnancy. ${ }^{30}$

3. Patients who have accepted the treatment of laparoscopic conservative operation;

4. Husband's semen analysis is normal;

5. Willingness to sign the consent form.

\section{Exclusion criteria}

Participants meeting any of the following criteria will be excluded:

1. Those who have other infertility factors (such as PCOS, tubal blockage, immune infertility, reproductive malignant tumor, reproductive organ abnormalities, and so on);

2. Those who have used other drugs that affect reproductive function or metabolism in the past 3 months;

3. Those who are not appropriate for acupuncture;

4. Those who have allergies to Chinese herbs or GnRH-a;

5. Patients with serious cardiovascular, liver, kidney, and hematopoietic system diseases;

6. Patients with infectious diseases, mental illness, and other medical histories.

\section{Interventions}

This study has two phases. The treatment group will accept three menstrual cycles of acupuncture and Chinese herb treatment, while the control group will accept the GnRH-a therapy for three menstrual cycles. The pregnancy guidance will be provided to both groups. The second phase will include 12 menstrual cycles of follow-up without treatment. 


\section{The treatment group}

The design of the treatment group is guided by the theory of TCM, including CHM and acupuncture.

\section{Chinese herb medicine}

The oral YZD granules are produced by Tian Jiang Pharmaceutical Co., Ltd. (Jiangyin, Jiangsu, China). The herbs in the prescription will be mixed, cooked, filtered, and pressure spray-dried to form granules. The granules are packaged into small single-dose sachets. Participants are given two sachets twice a day for three menstrual cycles, and each dose was dissolved in boiled warm water.

\section{Acupuncture}

The essential acupuncture points are selected as follows: CV4 (Guanyuan); SP10 (Xuehai) and SP6 (Sanyinjiao). The additional individualized acupuncture points are chosen by the acupuncturist according to the patterns of identification: (1) qi deficiency with blood stasis plus CV8 (Shenque); ST36 (Zusanli) and CV6 (Qihai). (2) kidney deficiency with blood stasis will add with bilateral KI3 (Taixi), KI6 (Zhaohai) and BL23 (Shenshu). (3) phlegm and blood stasis add ST40 (Fenglong) and SP9 (Yinlingquan). (Table 1) Disposable, stainless steel needles are punctured through the device and inserted 10-30 $\mathrm{mm}$ deep depending on location when participants are in the supine position. The needles are retained for $30 \mathrm{~min}$, manually lifted, thrust, and twisted to get the de-qi sensation. When qi arrives, the acupuncturist can feel tightness around the needle, and the patients can also feel such sensations as soreness, numbness, distention, and heaviness at the point. These sensations can radiate along the course of the channel. The whole acupuncture treatment is carried out by the same acupuncturist who has a license from the Ministry of Health of China and has clinically practiced for more than 10 years. Every acupuncture session is conducted once a day, twice a week, and it lasts for $20 \mathrm{~min}$. The treatment is suspended during the menstrual period. If participants get pregnant or have severe allergies during treatment, the treatment is also be stopped.

\section{Table 1}

Points Location

CV4(Guanyuan) On the lower abdomen, 3 B-cun inferior to the centre of the umbilicus, on the anterior median line. 


\begin{tabular}{|c|c|}
\hline SP10(Xuehai) & $\begin{array}{l}\text { On the anteromedial aspect of the thigh, on the bulge of } \\
\text { the vastus medialis muscle, } 2 \text { B-cun superior to the medial } \\
\text { end of the base of the patella. }\end{array}$ \\
\hline SP6(Sanyinjiao) & $\begin{array}{l}\text { On the tibial aspect of the leg, posterior to the medial } \\
\text { border of the tibia, } 3 \text { B-cun superior to the prominence of } \\
\text { the medial malleolus. }\end{array}$ \\
\hline \multicolumn{2}{|l|}{ Syndrome1 } \\
\hline CV8(Shenque) & On the upper abdomen, in the centre of the umbilicus \\
\hline ST36(Zusanli) & $\begin{array}{l}\text { On the anterior aspect of the leg, on the line connecting } \\
\text { ST35 with ST41, } 3 \text { B-cun inferior to ST35. }\end{array}$ \\
\hline CV6(Qihai) & $\begin{array}{l}\text { On the lower abdomen, } 1.5 \text { B-cun inferior to the centre of } \\
\text { the umbilicus, on the anterior median line. }\end{array}$ \\
\hline \multicolumn{2}{|l|}{ Syndrome2 } \\
\hline KI3(Taixi) & $\begin{array}{l}\text { On the posteromedial aspect of the ankle, in the depression } \\
\text { between the prominence of the medial malleolus and the } \\
\text { calcaneal tendon }\end{array}$ \\
\hline KI6(Zhaohai) & $\begin{array}{l}\text { On the medial aspect of the foot, } 1 \mathrm{~B} \text {-cun inferior to } \mathrm{KI} 3 \text {, } \\
\text { in the depression anterior to the calcaneal tuberosity. }\end{array}$ \\
\hline BL23(Shenshu) & $\begin{array}{l}\text { In the lumber region, at the same level as the inferior } \\
\text { border of the spinous process of the second lumbar } \\
\text { vertebra(L2), } 1.5 \text { B-cun lateral to the posterior median } \\
\text { line. }\end{array}$ \\
\hline \multicolumn{2}{|l|}{ Syndrome3 } \\
\hline ST40(Fenglong) & $\begin{array}{l}\text { On the anterolateral aspect of the leg, lateral border of the } \\
\text { tibialis anterior muscle, } 8 \text { B-cun superior to the } \\
\text { prominence of the lateral malleolus. }\end{array}$ \\
\hline SP9(Yinlingquan) & $\begin{array}{l}\text { On the tibial aspect of the leg, in the depression between } \\
\text { the inferior border of the medial condyle of the tibia and } \\
\text { the medial border of the tibia. }\end{array}$ \\
\hline
\end{tabular}

\section{The control group}

As the GnRH-a therapy after surgery is considered the 'first-line' treatment in moderate and severe endometriosis, and associated infertilit, ${ }^{12}$ the control group is 
subjected to the treatment of GnRH-a. Triptorelin for injection made by Ipsen Pharma Biotech is used in the present study, and $3.75 \mathrm{mg}$ once a menstrual cycle is given by intramuscular injection. The treatment lasts for three menstrual cycles.

\section{Outcome measurements}

Outcomes are determined at baseline, after three menstrual cycles of treatment, and follow-up at 3,6 and 12 menstrual cycles after the completion of the treatment. Table 2 lists the overview of outcome measurements at the different time points.

Table 2

\begin{tabular}{|c|c|c|c|c|}
\hline & $\begin{array}{l}\text { Screening } \\
\text { and baseline } \\
\text { visit }\end{array}$ & $\begin{array}{l}\text { After the } \\
\text { last } \\
\text { treatment }\end{array}$ & $\begin{array}{l}\text { Follow-up periods } \\
(3,6,12 \text { menstrual } \\
\text { cycles })\end{array}$ & $\begin{array}{l}\text { Any time（If } \\
\text { participants get } \\
\text { pregnancy） }\end{array}$ \\
\hline $\begin{array}{l}\text { General } \\
\text { condition }^{\mathrm{a}}\end{array}$ & $\sqrt{ }$ & & & \\
\hline $\begin{array}{l}\text { Gynecological } \\
\text { examination }\end{array}$ & $\sqrt{ }$ & & & \\
\hline $\begin{array}{l}\text { Pregnancy } \\
\text { test (blood } \\
\beta-H C G)\end{array}$ & $\sqrt{ }$ & $\sqrt{ }$ & $\sqrt{ }$ & $\sqrt{ }$ \\
\hline
\end{tabular}

Fasting blood

samples for

CA125,

AMH, sex

hormones ${ }^{b}$

and ALT

\section{Transvaginal \\ ultrasound}

\section{Treatment}

record $^{\mathrm{c}}$

\section{Questionnaire}

d

$\sqrt{ } \quad \sqrt{ } \quad \sqrt{ }$

a General condition include age, endometriosis-related surgery history, EFI, r-AFS, menstrual and obstetrical histories 
b Sex hormones include: E2, T, P, FSH, LH, PRL

c Treatment record includes date and adverse events

d Questionnaire includes the EHP-5

\section{Outcome measurements}

\section{Primary outcomes}

\section{Pregnancy rate}

Pregnancy is observed through blood HCG and gestational sac examined by transvaginal ultrasound when the time is coming or some signs of possible pregnancy appear in 1 year.

\section{Secondary outcomes}

\section{Ultrasound findings}

Ultrasound is used to observe the uterus, ovary, and the condition of recurrence. These findings can prove the pregnancy, assess the possibility of pregnancy, and observe the situation of recurrence. Moreover, the size of the uterus and ovary, endometrium thickness, and the abnormal situation are recorded. The participants take ultrasound examinations during the ovulation cycle or any time if participants probably get pregnant.

\section{CA125, AMH, sex hormones, and ALT}

$\mathrm{CA} 125, \mathrm{AMH}$, and sex hormones reflect the condition of endometriosis, infertility, and ovarian function. The fasting blood samples are collected on the third day of menstruation by the nurse. The chemiluminescence immunoassay (CLIA) is used to determine the CA125 level and serum sex hormone levels, including estradiol (E2), testosterone $(\mathrm{T})$, progesterone $(\mathrm{P})$, follicle-stimulating hormone (FSH), luteinizing hormone (LH), and prolactin (PRL). Enzyme-linked immunosorbent assay (ELISA) is used to determine the AMH level. The ALT level is determined by an automatic biochemical analyzer. These tests are all performed by the Yueyang Hospital Medical Testing Center.

\section{Endometriosis-specific quality of life}

The Endometriosis Health Profile-5 (EHP-5) is used to evaluate the effects of endometriosis on the life of participants. The test includes two parts, with questions referring to the four previous weeks: a 5-item core questionnaire about pain, control and powerlessness, emotions, social support, and self-image, and a 6-item modular 
questionnaire about work-life, relation with children, sexual intercourse, the medical profession, treatment, and infertility. The response system consists of five levels in an order of severity: 'never', 'rarely', 'sometimes', 'often', and 'always'. 31

\section{Adverse events/serious adverse events (AEs/SAEs)}

AEs for each treatment are monitored during the trial, including discomfort, sweating, hematoma, fainting, severe pain, metal allergy symptoms, and damage to liver or kidney function. These above-mentioned AEs are recorded by the researcher. If any participant experiences an $\mathrm{AE}$ due to trial participation, they can receive free treatment and compensation accordingly. If concerns are identified during the study, the participants can withdraw from the trial at any time.

\section{Termination, withdrawal, and loss}

The trial is terminated if any participant develops one or more of the following conditions during the trial: (1) the participant gets pregnant; (2) intolerable side effects, or (3) serious acute or chronic organic disease. Any participant can withdraw from the trial for any reason at any time without prejudicing current or future treatments. The investigators will contact any participants who withdraw or terminate the treatment to complete the final assessment. All withdrawn and terminated cases are reported and analyzed. The possibility of loss to follow-up is considered and calculated as a part of the sample size estimation. The dropout rate and reasons are recorded. Besides, other types of randomly missing data are accounted for by treating dropouts as non-success or non-survival using the intention-to-treat principle.

\section{Sample size and randomization}

The sample size of the current trial is calculated based on the formula as follows ${ }^{32}$ :

$$
n_{1}=n_{2}=\left[\frac{\left(Z_{1-\alpha}+Z_{1-\beta}\right)}{P_{1}-P_{2}-\Delta}\right]^{2}\left[P_{1} \times\left(1-P_{1}\right)+P_{2} \times\left(1-P_{2}\right)\right]
$$

According to recently published clinical trial results, ${ }^{33,34}$ the pregnancy rates after the laparoscopic conservative operation, acupuncture, and CHM treatment reach $70 \%\left(\mathrm{P}_{1}\right)$ in the treatment group and $50 \%\left(\mathrm{P}_{2}\right)$ in the control group. By using the superiority test, the calculation equation is $\alpha=0.025,1-\beta=0.80, \Delta=0$. A sample size of 112 patients in each group is sufficient to detect the statistical difference between the two groups, allowing for a withdrawal rate of $20 \%$. Upon confirmation of 
eligibility and receipt of informed consent, the participants are randomly assigned to the treatment and control groups. An independent statistician will provide computer-generated random sequences through Statistical Product and Service Solutions (SPSS) software. The random sequence for group classification is sealed in opaque envelopes and numbered consecutively according to the rank sequence.

\section{Data collection and management}

The investigators in the research team are required to attend a training workshop before recruitment. Each member of the research team receives a copy of the study protocol, and they are asked to strictly follow the protocol during the study period. The investigators responsible for data collection are trained to report interpretation and EHP-5. The ultrasound examination and blood tests are conducted by medical professionals.

Data are collected and recorded on case report forms. All data are entered into a predesigned password-protected electronic data capture by personnel blinded to the group allocation. Data entry is continually performed throughout the trial using the double-entry method, with any corrections or changes of data written in the case report forms documented and dated.

This study can establish the Data and Safety Monitoring Board (DSMB), which is composed of three members, including an acupuncturist, a gynecologist, and a statistician. All members must declare any conflict of interest during the trial. The DSMB will monitor the progress of the trial and review the safety and quality of the data. They meet quarterly to review any AEs and safety issues. All AEs and SAEs are reported to the principal investigator and DSMB within $24 \mathrm{~h}$. The primary investigator receives the interim results and makes the final decision once the study is completed.

\section{Data analysis}

All statistical analyses are performed using the SPSS program V.25.0.

Measurement data: Data are expressed as mean $\pm \mathrm{SD}(\mathrm{x} \pm \mathrm{s})$ by using one-way ANOVA if the variables obey a normal distribution and homoscedasticity. Non-normally distributed variables are corrected or analyzed using the rank-sum test. Enumeration data: The chi-square test is used for normally distributed data. However, Fisher's exact test is used when the theoretical frequency of the single cell is $<1$. Non-normally distributed variables are corrected or analyzed using the rank-sum test. All hypothesis tests are performed using two-sided tests, with the test standard $\alpha=0.05$. $\mathrm{P}<0.05$ was considered statistically significant. 


\section{Discussion}

Endometriosis is a complex disease with no exact pathogenic factors. The mechanism underlying infertility in endometriosis also remains largely undetermined. It may exist in the pelvic cavity, ovary, or uterus. ${ }^{35}$ Meanwhile, the therapies are limited and under research. The common hormonal medications after surgery prevent women to be pregnant during treatment. Therefore, patients may miss the optimal time of pregnancy after surgery.

Although the effect of acupuncture and YZD on EAI is not clear, our previous research shows that acupuncture and CHM can effectively prevent the recurrence of endometriosis after surgery, and improve menstruation condition and life quality. ${ }^{36}$ However, the therapeutic effect still requires further rigorous scientific verification.

Currently, acupuncture or YZD has been proved to be efficient for the treatment of EAI. Relevant RCT studies do not combine acupuncture and YZD, do not follow the CONSORT or SPIRIT Statement, and are published in languages other than English. Therefore, large RCTs are required to assess the role and possible adverse outcomes of acupuncture and YZD for the treatment of EAI.

We combine TCM therapy with scientific and rigorous experimental design to explore the effects of acupuncture and YZD on EAI. The acupoints are selected based on the syndrome differentiation to adequately demonstrate the dialectical treatment of TCM. The results of this trial could provide high-quality evidence of the efficacy of acupuncture and YZD in increasing the pregnancy rate in infertile women with endometriosis.

\section{Patient and public involvement}

Patients' priorities, experience and preferences were not involved in the development of the research question and outcome measures, the design of this study, or the recruitment to and conduct of the study. The results will be disseminated to study participants by telephone or email interviews. All the expenses of the examinations and treatments will be borne by the research group.

\section{Trial status}

This protocol version number is 2.0, dated November 2020. Participant recruitment began in January 2021 and will end end in December 2021.

\section{Abbreviations}


IVF: In-vitro fertilization

TCM: Traditional Chinese Medicine, CHM Chinese herb medicine

YZD: Yangjing Zhongyu Decoction, DSMB Data and Safety Monitoring Board

EAI: Endometriosis-associated infertility

E2: Estradiol

$\mathrm{T}$ : Total testosterone

P: Progesterone

FSH: Follicle-stimulating hormone

LH: Luteinizing hormone

PRL: Prolactin

AMH: Anti-Müllerian hormone

EFI: Endometriosis fertility index

r-AFS: revised American Fertility Society classification

EHP-5: The Endometriosis Health Profile -5

\section{Acknowledgments}

Thanks to all the participants who provided valuable input to the trial and participated in the trial. We would also like to thank the staff of this trial from all hospitals, for their advice and support throughout the trial process.

\section{Authors' contributions}

T-cW and Y-cZ conceived the research plan, drafted the protocol and formed the analysis plan. Y-xZ coordinated the study. T-cW, Y-cZ and Y-xZ recruited the subjects. All authors participated in, read and approved the final manuscript.

\section{Availability of data and materials}

The data that support the findings of this study will be available from authors but restrictions apply to the availability of these data, which will be used under license for the current study, and so are not publicly available. Data will be available from the authors upon reasonable request.

\section{Competing interests}

The authors declare that they have no competing interests.

\section{Consent for publication}

Not applicable. Results will be presented at relevant national and international conferences as well as being published in peer-reviewed journals.

\section{Funding}


This trial is supported by innovation funds of Science and Technology Commission of Shanghai Municipality (STCSM), China (Project No.: 19401934500); Clinical Research Plan of SHDC (Project No.: SHDC2020CR3093B); National Natural Science Foundation of China (Project No. 81403429); Xinglin Scholar, Shanghai University of Traditional Chinese Medicine.

\section{Ethics approval and consent to participate}

This trial protocol is in accordance with the principles of the Declaration of Helsinki and has been approved by Ethics Committee of Yueyang Hospital of Integrated Traditional Chinese and Western Medicine (approval no.2020-025) (No. 2017-083-KY-01) on 30 November 2020, and was registered with Chinese Clinical Trial Registry (ChiCTR2100042830) on 29 January 2021(http://www.chictr.org.cn/). Written informed consent will be obtained from all participants. The results of this study will be disseminated to the public through academic conferences and peer-reviewed journals.

\section{Author details}

1.Yueyang Hospital of Integrated Traditional Chinese and Western Medicine, Shanghai University of Traditional Chinese Medicine, Shanghai, China

2.Shanghai University of Traditional Chinese Medicine, Shanghai, China

3.Longhua Hospital Shanghai University of Traditional Chinese Medicine, Shanghai, China

\section{References}

1 Johnson NP, Hummelshoj L; World Endometriosis Society Montpellier Consortium. Consensus on current management of endometriosis. Hum Reprod. 2013;28(6):1552-68.

2 Ballard KD, Seaman HE, de Vries CS, Wright JT. Can symptomatology help in the diagnosis of endometriosis? Findings from a national case-control study--Part 1. BJOG. 2008;115(11):1382-91.

3 Meuleman C, Vandenabeele B, Fieuws S, Spiessens C, Timmerman D, D'Hooghe T. High prevalence of endometriosis in infertile women with normal ovulation and normospermic partners. Fertil Steril. 2009;92(1):68-74.

4 Missmer SA, Hankinson SE, Spiegelman D, Barbieri RL, Marshall LM, Hunter DJ. Incidence of laparoscopically confirmed endometriosis by demographic, anthropometric, and lifestyle factors. Am J Epidemiol. 2004;160(8):784-96. 
5 Buyalos RP, Agarwal SK. Endometriosis-associated infertility. Curr Opin Obstet Gynecol. 2000;12(5):377-81.

6 Dunselman GA, Vermeulen N, Becker C, Calhaz-Jorge C, D'Hooghe T, De Bie B, et al. ESHRE guideline: management of women with endometriosis. Hum Reprod. 2014;29(3):400-12.

7 Duffy JM, Arambage K, Correa FJ, Olive D, Farquhar C, Garry R, et al. Laparoscopic surgery for endometriosis. Cochrane Database Syst Rev. 2014;(4):CD011031. Published 2014 Apr 3.

8 Hornstein MD, Hemmings R, Yuzpe AA, Heinrichs WL. Use of nafarelin versus placebo after reductive laparoscopic surgery for endometriosis. Fertil Steril. 1997;68(5):860-4.

9 Macer ML, Taylor HS. Endometriosis and infertility: a review of the pathogenesis and treatment of endometriosis-associated infertility. Obstet Gynecol Clin North Am. 2012;39(4):535-49.

10 Practice Committee of the American Society for Reproductive Medicine. Endometriosis and infertility: a committee opinion. Fertil Steril. 2012;98(3):591-8. 11 Audebert A, Descamps P, Marret H, Ory-Lavollee L, Bailleul F, Hamamah S. Pre or post-operative medical treatment with nafarelin in stage III-IV endometriosis: a French multicenter study. Eur J Obstet Gynecol Reprod Biol. 1998;79(2):145-8. 12 Sõritsa D, Saare M, Laisk-Podar T, Peters M, Sõritsa A, Matt K, et al. Pregnancy rate in endometriosis patients according to the severity of the disease after using a combined approach of laparoscopy, GnRH agonist treatment and in vitro fertilization. Gynecol Obstet Invest. 2015;79(1):34-9.

13 Barri PN, Coroleu B, Tur R, Barri-Soldevila PN, Rodríguez I.

Endometriosis-associated infertility: surgery and IVF, a comprehensive therapeutic approach. Reprod Biomed Online. 2010;21(2):179-85.

14 Burke A, Upchurch DM, Dye C, Chyu L. Acupuncture use in the United States: findings from the National Health Interview Survey. J Altern Complement Med. 2006;12(7):639-48.

15 Bovey M, Lorenc A, Robinson N. Extent of acupuncture practice for infertility in the United Kingdom: experiences and perceptions of the practitioners. Fertil Steril. 2010;94(7):2569-73. 
16 Gao R, Guo B, Bai J, Wu Y, Wu K. Acupuncture and clomiphene citrate for anovulatory infertility: a systematic review and meta-analysis. Acupunct Med. 2020;38(1):25-36.

$17 \mathrm{Wu}$ L, Wei DH. Effect of laparoscopic surgery combined with TCM treatment on the symptoms and pregnancy outcomes in patients with endometriosis and infertility. Clinical Research and Practice. 2018;3(02):54-5.

18 Shi JM, Zhu Y. Effect of Traditional Chinese Medicine for Kidney-tonifying, Blood-activating and Stasis-removing combined with laparoscopic surgery on sex hormone, oxidative stress and fertility of infertile patients with endometriosis. Maternal and Child Health Care of China. 2019;34(24):5740-2.

19 Liu TT, Wu J, Zhang ZH, Liang RN. Meta-analysis of traditional Chinese medicine combined with acupuncture in the treatment of PCOS infertility. Electronic Journal of Practical Gynecologic Endocrinology. 2019;6(19):95-8.

20 Wang LW, Hu QL, Huang LJ, Liu BP. Meta-analysis of acupuncture combined with medicine in the treatment of immune infertility. Asia-Pacific Traditional Medicine. 2017;13(04):66-9.

21 Ma HX, Xie J, Lai MH, Zheng YH, Liu H, Ding T, et al. Effects of Yangjing Zhongyu Decoction on the Secretion of Ovarian Granule Cells in Polycystic Ovarian Syndrome Rat Model. Chinese Journal of Integrated Traditional and Western Medicine. 2012;32(01):54-7.

22 Duan H, Luo HL. Effect of Yangjing Zhongyu Decoction on the quality of mouse oocytes and its relationship with ovarian EGF. Lishizhen Medicine and Materia Medica Research. 2015;26(09):2123-5.

23 Zhang L, Zhang JW, Wu HC. Effects of Yangjing Zhongyu Decoction on endometrial receptivity in deficiency of kidney yin patients with repeated implantation failure. China Journal of Traditional Chinese Medicine and Pharmacy. 2019;34(08):3842-5.

24 Zhang YX, Zhang Q, Wang JR, Li MY, Liu P, Han XY. Effects of Yangjing Zhongyu Decoction Assisted in Vitro Fertilization embryo Transfer on the Patients with Ovulatory Disorder Infertility. World Chinese Medicine. 2020;15(18):2743-6. 25 Wang X, Wu WC. An analysis of mechanism of treating female infertility with the Yangjing Zhongyu decoction. Clinical Journal of Chinese Medicine. 2017;9(11):122-3. 
26 Cheng CW, Wu TX, Shang HC, Li YP, Altman DG, Moher D, Bian ZX; CONSORT-CHM Formulas 2017 Group. CONSORT Extension for Chinese herbal medicine formulas 2017: recommendations, explanation, and elaboration (Traditional Chinese Version). Ann Intern Med. 2017;167:W7-20.

27 MacPherson H, Altman DG, Hammerschlag R, Youping L, Taixiang W, White A, et al. Revised STandards for Reporting Interventions in Clinical Trials of Acupuncture (STRICTA): extending the CONSORT statement. PLoS Med. 2010;7(6):e1000261.

28 Chan AW, Tetzlaff JM, Altman DG, Laupacis A, Gøtzsche PC, Krleža-Jerić K, et al. SPIRIT 2013 statement: defining standard protocol items for clinical trials. Ann Intern Med. 2013;158(3):200-7.

29 Revised American Society for Reproductive Medicine classification of endometriosis: 1996. Fertil Steril. 1997;67(5):817-21.

30 Practice Committee of the American Society for Reproductive Medicine.

Definitions of infertility and recurrent pregnancy loss: a committee opinion. Fertil Steril. 2013;99(1):63.

31 Jones G, Jenkinson C, Kennedy S. Development of the Short Form Endometriosis Health Profile Questionnaire: the EHP-5. Qual Life Res. 2004;13(3):695-704.

32 Deng W, He J. Design and statistically analysis of clinical trials. Beijing: People's Medical Publishing House; 2012. p. 141-142.

33 Li FY, Zhang CY, Li YB, Jiang DS, Yang YL. Effect of Traditional Chinese Medicine for Blood-activating and Stasis-removing Combined with Acupuncture and Moxibustion on Serum CA-125 Level, EMAb and Pregnancy Outcome in Patients with Endometriosis Complicated with Infertility. Hubei Journal of Traditional Chinese Medicine. 2018;40(12):15-8.

34 Jin X, Ruiz Beguerie J. Laparoscopic surgery for subfertility related to endometriosis: a meta-analysis. Taiwan J Obstet Gynecol. 2014;53(3):303-8. 35 de Ziegler D, Borghese B, Chapron C. Endometriosis and infertility: pathophysiology and management. Lancet. 2010;376(9742):730-8.

36 Zhang C, Zhang X, Li L, Zhou Y. Clinical effect evaluation of acupuncture combined with medication for prevention of endometriosis recurrence after surgery. Zhongguo Zhen Jiu. 2016;36(3):237-42. 


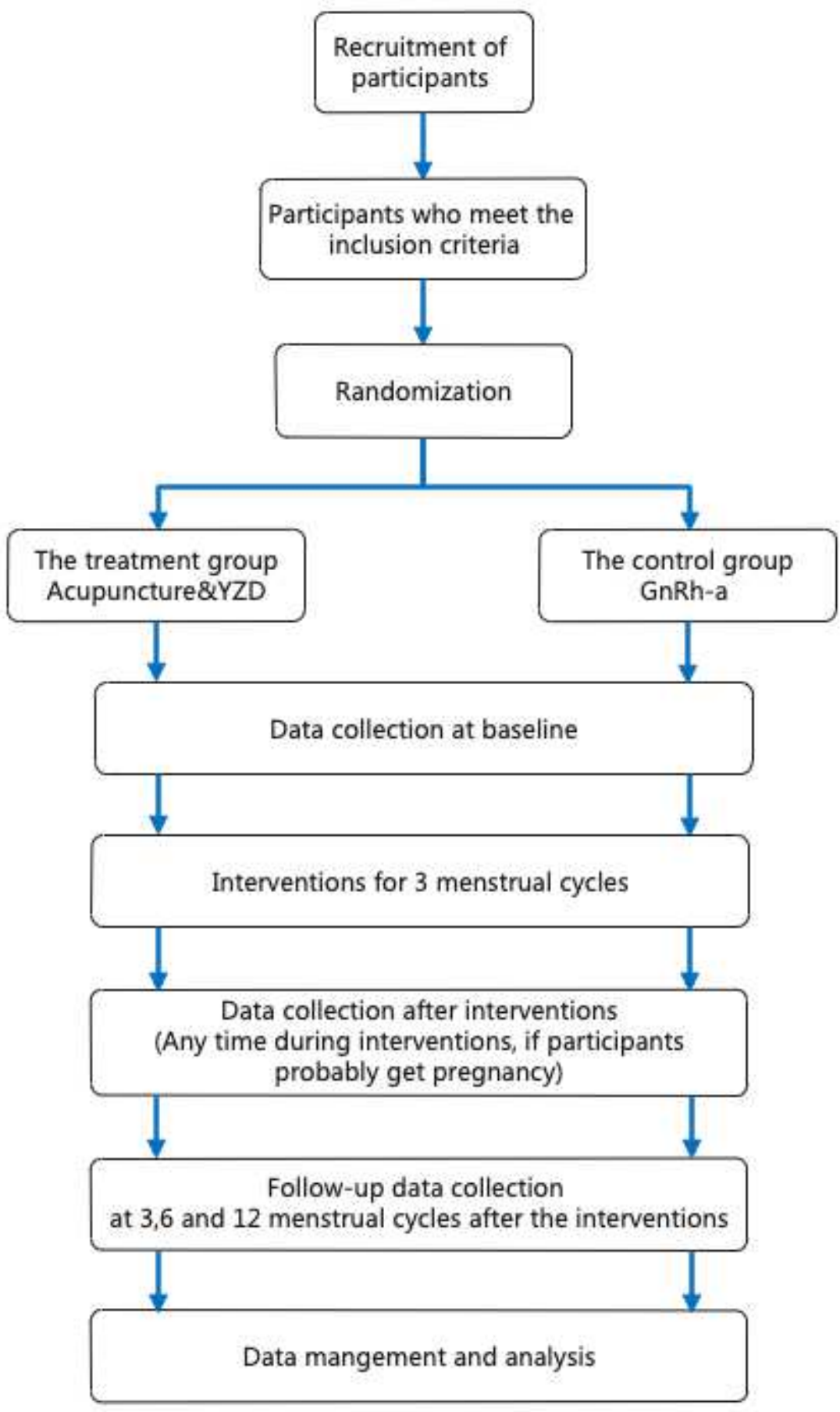

\section{Figure 1}

The flow chart.

\section{Supplementary Files}

This is a list of supplementary files associated with this preprint. Click to download. 
- SPIRITchecklist.pdf

- APPENDIX12.pdf 